\title{
Spatial Resolution Test of a Beam Profile Monitoring System for the Double Electrostatic Ion Storage Ring
}

\author{
S. DAS ${ }^{* \dagger}$ AND A. KÄLLBERG \\ Manne Siegbahn Laboratory, Department of Physics, Stockholm University, Stockholm, SE-10691, Sweden \\ (Received August 23, 2012; in final form August 30, 2013)

\begin{abstract}
A beam profile monitoring system based on the imaging of low energy electrons created when the ion beam impinges on a metal plate was built to monitor the beam profile and to cover the wide range of beam intensities and energies for the DESIREE (Double ElectroStatic Ion Ring ExpEriment) beam line diagnostics. The spatial resolution of the system was tested with different beams of various energies and was found to be around $2 \mathrm{~mm}$. A significant steering effect on the ion beam before hitting the metallic foil was observed at low energies.
\end{abstract}

DOI: $10.12693 /$ APhysPolA.125.13

PACS: 07.05.Pj, 07.77.Ka, 07.05.Fb

\section{Introduction}

In recent years, single electrostatic ion storage rings, owing to their fundamental and technical advantages over magnetic storage rings, have attracted considerable attention among the scientists [1-7]. Motivated by the success of electrostatic ion storage rings and the possibility of performing merged-beams experiments with positive and negative ions, Double ElectroStatic Ion Ring ExpEriment (DESIREE) as shown in Fig. 1 has been constructed at Stockholm University and will soon be ready for the experiments [8]. As the name implies, it consists of two electrostatic storage rings (each of circumference $8.8 \mathrm{~m}$ ) with a common straight section (length of $1 \mathrm{~m}$ ) where merged-beams experiments on positive/negative ion collisions can be performed. The two rings are housed in a single double-walled vacuum chamber built like a cryostat with a radiation screen and several layers of insulation in between the two chambers. The inner chamber, which holds all the optical elements, is cooled by cryogenerators attached to the bottom of this chamber so that the entire structure can be cooled to temperatures below $15 \mathrm{~K}$. Two high voltage platforms (injectors) $100 \mathrm{kV}$ and $25 \mathrm{kV}$, respectively, are used to create the ion beams for measurements. Ion beams of opposite charges can be stored in the two rings where neutral and charged products of collisions between the oppositely charged ion beams can be observed after the straight merging section. Furthermore, each ring can be envisioned for experiments with a single stored ion beam interacting with pulsed or continuous (cw) laser beams. Thus, the low temperature in combination with the double ring structure will make DESIREE a unique machine to perform both fun-

*Present address: Institute for Laser Science, University of Electro-Communications, 1-5-1 Chofugaoka, Chofu, Tokyo-182-8585, Japan

${ }^{\dagger}$ corresponding author; e-mail: das@ils.uec.ac.jp damental and applied research. The detailed description of DESIREE with all the parameters is given in Ref. [8].

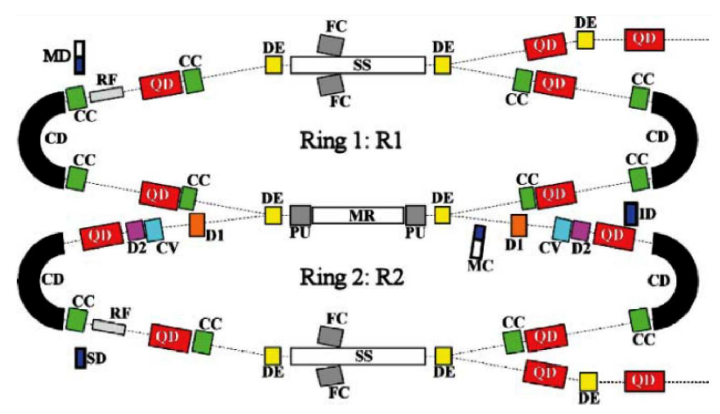

Fig. 1. Schematic of ion-optical structure of DESIREE. $\mathrm{R} 1$ and $\mathrm{R} 2$ are two storage rings $(\mathrm{R} 1=\mathrm{R} 2=$ $8.8 \mathrm{~m}), \mathrm{QD}$ - quadrupole doublets, $\mathrm{CC}$ and $\mathrm{CV}$ correction elements, $\mathrm{CD}-160^{\circ}$ deflector, $\mathrm{DE}-10^{\circ}$ deflector, D1 and D2 - deflector, FC - Faraday cup, $\mathrm{PU}$ - pick-up, ID — imaging detector, MD - movable neutral detector, SD - stationary neutral detector, $\mathrm{MC}$ - movable charged fragment detector, RF - radio frequency kicker, SS and MR (1 m) — straight and merging sector, respectively (see Ref. [8] for more detail).

For use in the DESIREE beam line, between the high voltage platform and DESIREE, a beam profile monitoring system (BPMS) was built and tested at the CRYRING (CRYogenic Stockholm ion source-synchrotron storage RING) facility at the Manne Siegbahn Laboratory, Stockholm University [6]. The BPMS was built according to the concept developed by Kruglov and his coworkers for radioactive beam experiments (REX). The motivation for choosing this type of monitor was its ability to monitor extremely low intensity beams and at the same time having a very large dynamic range. The principle is based on the imaging of low energy secondary electrons (SE) generated when the ion beam impinges on a metal plate [9-12].

Apart from BPMS, several other detectors and measuring devices such as the Faraday cups, electrostatic pick- 
ups etc., will also be mounted in the DESIREE [5]. It should be noted that in addition to the DESIREE beam line diagnostics, the BPMS was also used for tests of the new extraction at the CRYRING [13].

\section{Experimental setup}

To test the spatial resolution of the BPMS as well as its behavior with very low energy beams an experimental setup as shown in Fig. 2 was built. The BPMS consists of an aluminum (Al) plate, a grid placed in front of $\mathrm{Al}$, a double microchannel plate assembly (MCP), a phosphor screen (PS), a computer (PC), and a CCD camera. A beam collimator containing a set of circular holes of different diameters $(\varnothing)$ and separation $(d)$ between them was placed $\approx 27 \mathrm{~cm}$ in front of the BPMS. For the present measurements the two holes of diameter $1 \mathrm{~mm}$ each and separated by a distance of $2 \mathrm{~mm}$ in the collimator, and proton $(10 \mathrm{keV})$ and $\mathrm{H}_{2}^{+}(3.5,5,10$, and $20 \mathrm{keV})$ beams, respectively, were used.

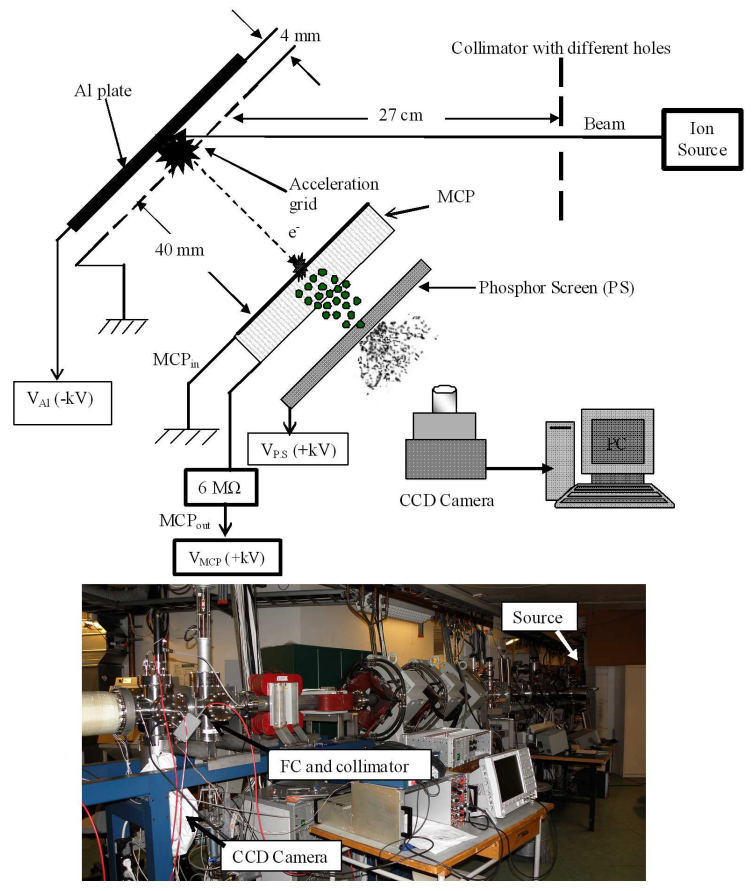

Fig. 2. (Upper part) Schematic diagram of the beam diagnostic setup and (lower part) experimental setup at the CRYRING facility.

A schematic drawing of the experimental setup is shown in Fig. 2.

The 3D drawing of the BPMS and drawing of the collimator are shown in Fig. 3. The collimator cuts out areas from the beam equal to the holes with separation $d \mathrm{~mm}$ between the beams centers, and creates well separated (distinguishable) narrow beams of approximately same intensity close to each other. Both the collimator and the Al plate attached with the grid can be inserted and taken out of the beam path by pneumatic feed-throughs.
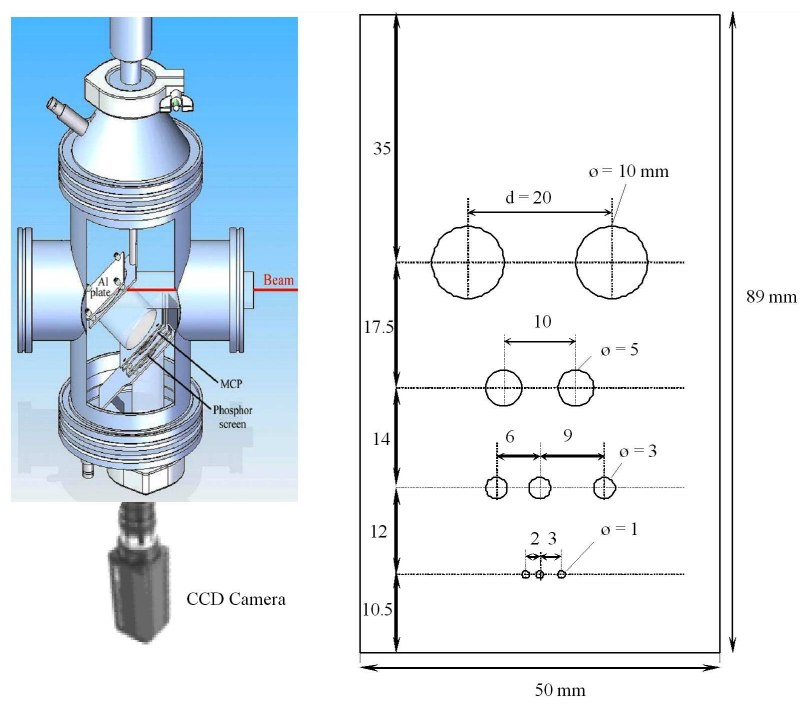

Fig. 3. (Left part) 3D view of the BPMS. (Right part) The collimator. It contains a set of circular holes of different diameters $(\varnothing)$ and separation $(d)$ between them. For the present measurement the bottom holes of diameter $1 \mathrm{~mm}$ each, separated by $2 \mathrm{~mm}$ were used.

The beam from the ion source passes through the collimator, strikes the Al plate, and knocks off low energy SE. The SE are accelerated by a homogeneous electric field applied between the Al plate and the grid, kept at negative and ground potentials, respectively. The accelerated electrons from the grid then travel the field free region to the MCP where they are amplified. After leaving from the back of the MCP, the cascade of electrons hits the PS and produces flashes of light, which, in turn, are captured by a CCD camera. This camera is connected to a $\mathrm{PC}$ for further storage and analysis of the recorded light intensities [14].

\section{Results and discussion}

CCD images of the light produced in the PS after the beam passing through the collimator and striking the $\mathrm{Al}$ plate are shown in Fig. 4 for $\mathrm{H}_{2}^{+}$and proton beams for different Al plate $\left(V_{\mathrm{Al}}\right)$, MCP $\left(V_{\mathrm{MCP}}\right)$, and PS $\left(V_{\mathrm{PS}}\right)$ voltages. The upper panel is for $3.5 \mathrm{keV} \mathrm{H}_{2}^{+}$beam for different $V_{\mathrm{PS}}$ at fixed $V_{\mathrm{MCP}}(1326 \mathrm{~V})$ and $V_{\mathrm{Al}}(-6.4 \mathrm{kV})$, middle and lower panels are for $10 \mathrm{keV}$ proton beam for different $V_{\mathrm{Al}}$ at fixed $V_{\mathrm{MCP}}(1370 \mathrm{~V})$ and $V_{\mathrm{PS}}(4000 \mathrm{~V})$, and for different $V_{\mathrm{MCP}}$ at fixed $V_{\mathrm{Al}}(-4.9 \mathrm{kV})$ and $V_{\mathrm{PS}}$ $(4600 \mathrm{~V})$, respectively.

Figure 5 shows the corresponding horizontal image profiles as a function of pixel (distance) for $3.5 \mathrm{keV} \mathrm{H}+$ beam. From the plot, it was found that 20 pixels correspond to $1 \mathrm{~mm}$. Two beam spots of diameter $1 \mathrm{~mm}$, separated by $2 \mathrm{~mm}$ are clearly seen (upper spots) from Fig. 4. This suggests a spatial resolution of $2 \mathrm{~mm}$ of the system. The diffused lower spots in the images are unaffected by the detector voltages as well as any changes in the focusing 

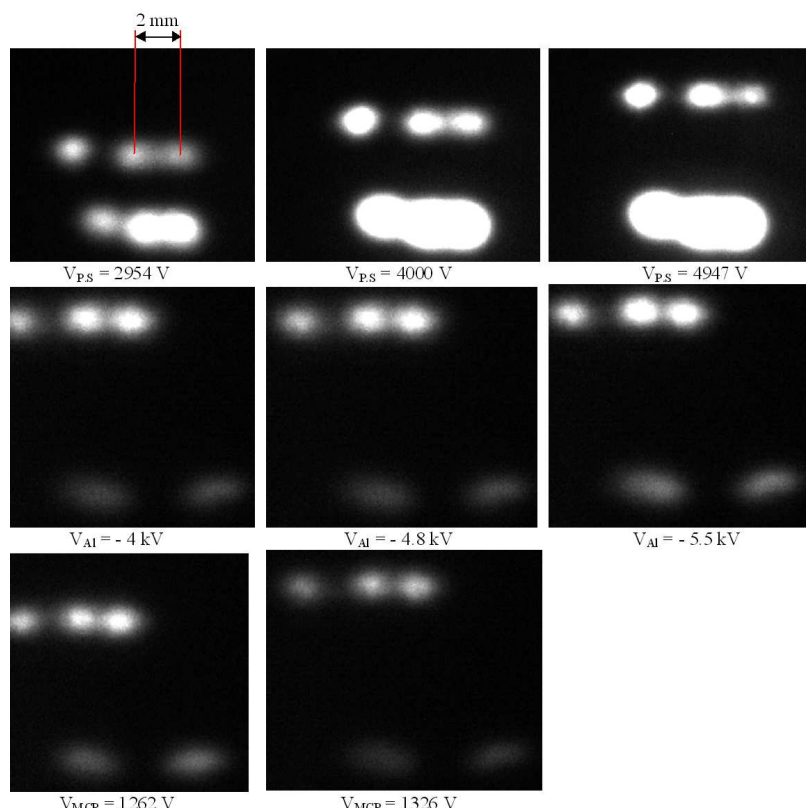

Fig. 4. CCD images (upper spots) of the light produced in PS by $\mathrm{H}_{2}^{+}$and proton beams of different energies. The upper part is for $3.5 \mathrm{keV} \mathrm{H}_{2}^{+}$beam for different $V_{\mathrm{PS}}$ at fixed $V_{\mathrm{MCP}}(1326 \mathrm{~V})$ and $V_{\mathrm{Al}}(-6.4 \mathrm{kV})$, middle part is for $10 \mathrm{keV}$ proton beam for different $V_{\mathrm{Al}}$ at fixed $V_{\mathrm{MCP}}(1370 \mathrm{~V})$ and $V_{\mathrm{PS}}(4000 \mathrm{~V})$, and lower part is for $10 \mathrm{keV}$ proton beam for different $V_{\mathrm{MCP}}$ at fixed $V_{\mathrm{PS}}(4600 \mathrm{~V})$ and $V_{\mathrm{Al}}(-4.9 \mathrm{kV})$, respectively. Two beam spots of diameter $1 \mathrm{~mm}$ each and separated by $2 \mathrm{~mm}$ are clearly seen, suggesting a spatial resolution of $2 \mathrm{~mm}$ of the BPMS. The diffused lower spots in the images, used as reference, are probably due to the Bremssstrahlung photons that are created when the beam passes through the narrow channel of the RFQ. In the upper part, the photon images are saturated since a high gain was needed to see the images of the relatively weak ion beam. The images of the ions (upper spots in the images) shift relative to the photon (reference) images. (Scale: 20 pixels correspond to $1 \mathrm{~mm}$ ).

elements in the beam line before the detector. While these spots can be troublesome by causing a background to the ion spots, in this experiment they were also an advantage, since they constituted a fixed position reference on the screen. It can also be seen from Fig. 4 that the images of the circular holes (upper spots) shift with respect to the diffused images (lower spots). The shift of the beam spots from the reference image was investigated for the $3.5 \mathrm{keV} \mathrm{H}_{2}^{+}$beam and found to be increasing with increasing $V_{\mathrm{PS}}$ at fixed $V_{\mathrm{Al}}$ and $V_{\mathrm{MCP}}$ as shown in Fig. 6 .

The exact reason for the formation of these diffused spots for low energy beam is unknown but it can be suggested that they are caused by Bremsstrahlung photons that have been created when the beam passes through the narrow channel of the radio frequency quadruple (RFQ). During these tests, no voltage was applied to the RFQ, which thus acted as a long $(\approx 1 \mathrm{~m})$ and narrow $(\Phi \leq 10 \mathrm{~mm}, \Phi$ is diameter of the channel through

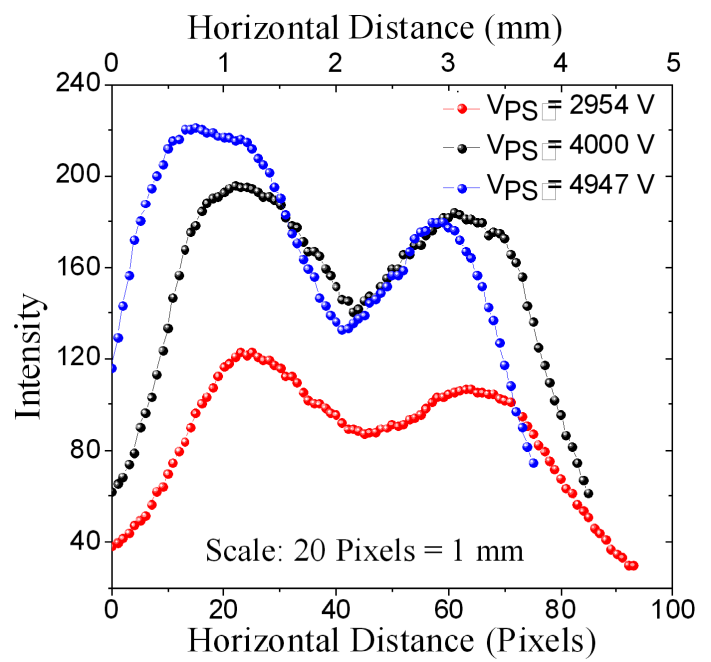

Fig. 5. Horizontal beam profiles of the CCD images produced on the phosphor screen.

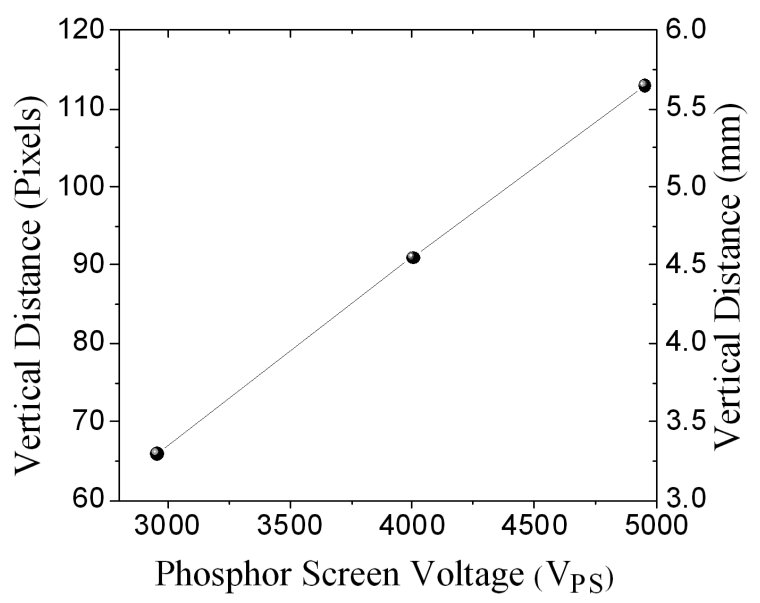

Fig. 6. Vertical shift of the CCD images from the diffused (reference) images as a function of $V_{\mathrm{PS}}$ for $3.5 \mathrm{keV}$ $\mathrm{H}_{2}^{+}$beam (see also upper part of Fig. 4).

RFQ) non-focussing channel. Therefore, a rather large part of the ion beam may have hit the copper rods inside the RFQ. It should be noted that no diffused spots were observed on the PS for $20 \mathrm{keV} \mathrm{H} \mathrm{H}_{2}^{+}$beam. This should be due to the fact that for higher energy the beam from the ion source is more focused and thus easier to transport through the RFQ without losses. Attempts were made to see even lower-energy beams on the monitor. However, no images were observed for $\mathrm{H}_{2}^{+}$beams below $3.5 \mathrm{keV}$.

Simulations with SIMION were performed to check the steering effect (influence of $V_{\mathrm{Al}}$ and $V_{\mathrm{PS}}$ ) on low/high-energy beams [15]. Results from these calculations are presented in Figs. 7 and 8, respectively. Figure 7 displays the impact of the electrical fields resulting from the voltages from the part of the detector with the MCP and the phosphor screen and the $\mathrm{Al}$ plate on the beam (proton 
or $\mathrm{H}_{2}^{+}$beam) trajectories for fixed $V_{\mathrm{MCP}}$ and $V_{\mathrm{PS}}$. To separate the effect of the fields from the two parts of the detector, the plate voltage was set to $0 \mathrm{~V}$ in the simulation shown in the upper part in the figure and $-6 \mathrm{kV}$ in the lower part. As can be seen, the low energy beam is strongly affected, both by the voltage applied on the $\mathrm{MCP} /$ phosphor screen package and by that of the plate. For very low beam energies, the deflection is even large enough to make the beam miss the Al plate.

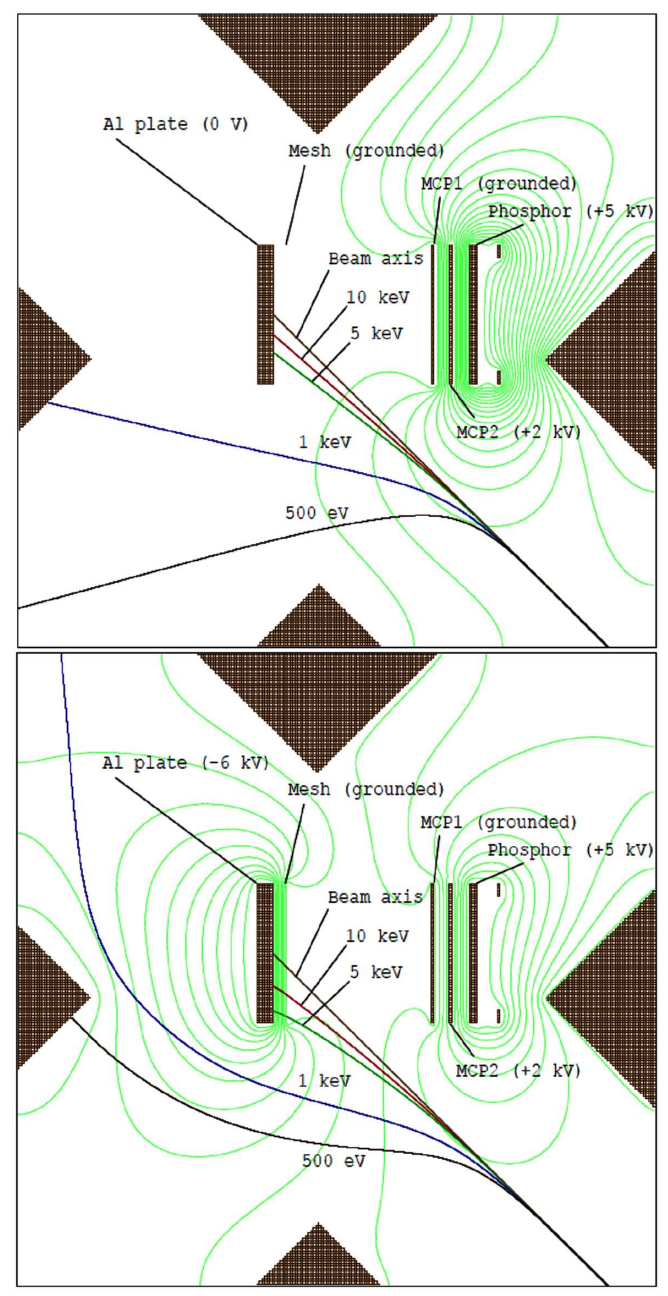

Fig. 7. Influence on the beam trajectories by the fields from the $\mathrm{MCP} /$ phosphor screen package and the $\mathrm{Al}$ plate voltages (upper part: $V_{\mathrm{Al}}=0 \mathrm{~V}$, lower part: $V_{\mathrm{Al}}=-6 \mathrm{kV}$ ). Green lines represent the electric field lines.

Figure 8 shows the results for $3.5 \mathrm{keV} \mathrm{H} \mathrm{H}_{2}^{+}$beam for 3 and $5 \mathrm{kV}$ phosphor screen voltages and the difference between the two points at which the beam hits the $\mathrm{Al}$ plate was found to be around $4 \mathrm{~mm}$. The displacement found in the simulation is about twice as large as the one we observed experimentally.

One reason for the stronger effect in the simulations could be that the beam trajectory in the simulations was chosen to have a downward angle after the collimator, in

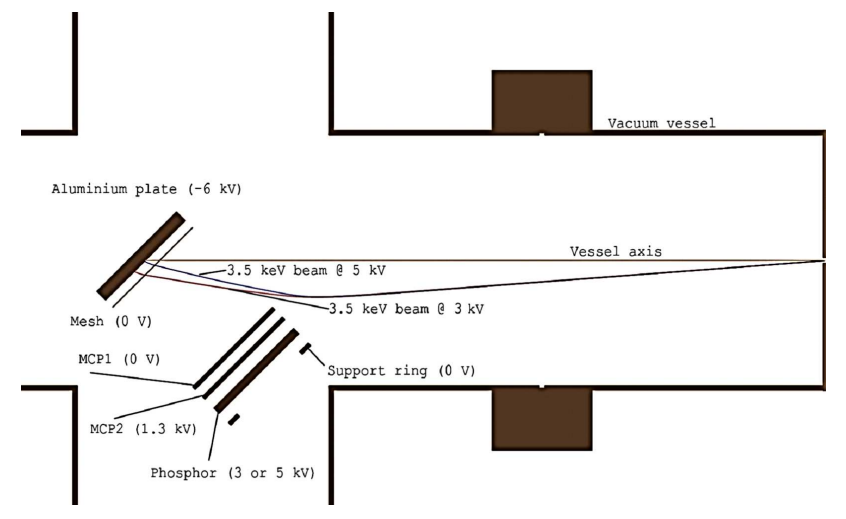

Fig. 8. Path of the $3.5 \mathrm{keV} \mathrm{H} \mathrm{H}_{2}^{+}$beams for different $V_{\mathrm{PS}}$. The incident beam was chosen to have a downward angle such that it hits the center of the Al plate for $5 \mathrm{kV}$ phosphor screen voltage. For $3 \mathrm{kV}$, the beam hits $4 \mathrm{~mm}$ below the center of the plate.

order for the beam to hit the center of the plate for the case with $5 \mathrm{kV}$ on the phosphor screen. This choice brings the beam closer to the MCP and phosphor screen unit and thus it is affected more strongly by the electric field from this part of the detector. Further simulations would be required to establish how much the exit angle affects the sensitivity of the electric field. The trajectories of the secondary electrons are not included in the simulations, since these mainly pass the field-free region between the grid and the MCPs and consequently are unaffected by the energy of the incoming beam. This view is supported by the fact that we were not able to observe any difference in resolution depending on beam energy.

To be able to use the monitor with lower-energy beams, a modified version of the detector would need to be constructed, in which grounded metal shields would be incorporated to shield the beam from the electrical fields of the MCP and the fluorescent screen. However, such a design would require more detailed simulations as well as further systematic investigations.

\section{Conclusions}

In summary, a beam profile monitoring system was built. The spatial resolution of the system was tested using a collimator and different beams of various energies. A resolution of $2 \mathrm{~mm}$ was achieved for all the energies. Two sets of images, real (upper spots) and reference (lower spots), were produced on the phosphor screen. Due to the effect of the electrical fields in the beam monitor on the ion beam trajectories, the real images shift with respect to these reference images. However, further detailed and systematic investigations would be needed to understand completely the underlying mechanism responsible for all these effects. There is a low energy limit for the use of this design of the monitor which is around $3 \mathrm{keV}$ for an $\mathrm{H}_{2}^{+}$beam. The experimental results were supported by the simulations. 


\section{Acknowledgments}

Dr. Das' participation in this research project was funded by the European Commission within FP7 Marie Curie Initial Training Network DITANET. The authors also thank Mr. Janusz Harasimowicz of the University of Liverpool, UK for making the SIMION calculations and for fruitful discussion in preparing the manuscript.

\section{References}

[1] S.P. Møller, Nucl. Instrum. Methods Phys. Res. A 394, 281 (1997).

[2] T. Tanabe, K. Chida, K. Noda, I. Watanabe, Nucl. Instrum. Methods Phys. Res. A 482, 595 (2002); T. Tanabe, K. Noda, Nucl. Instrum. Methods Phys. Res. A 496, 233 (2003); K. Noda, T. Tanabe, K. Noda, E. Syresin, Nucl. Instrum. Methods Phys. Res. A 532, 105 (2004).

[3] C.P. Welsch, J. Ullrich, C. Gläßner, A. Schempp, R. Dörner, H. Schmidt-Böcking, Nucl. Instrum. Methods Phys. Res. A 527, 284 (2004).

[4] D. Zajfman, A. Wolf, D. Schwalm, D.A. Orlov, M. Grieser, R. von Hahn, C.P. Welsch, J.R. Crespo Lopez-Urrutia, C.D. Schröter, X. Urbain, J. Ullrich, J. Phys., Conf. Ser. 4, 296 (2005).

[5] The CRYRING Accelerator facility, Manne Siegbahn Laboratory, Department of Physics, Stockholm University, Stockholm, Sweden, www.ms l.se .

[6] R.D. Thomas, Mass Spectrom. Rev. 27, 485 (2008).

[7] S. Schippers, Nucl. Instrum. Methods Phys. Res. B 267, 192 (2009).

[8] R.D. Thomas, H.T. Schmidt, G. Andler, M. Björkhage, M. Blom, L. Brännholm, E. Bäckström, H. Danared, S. Das, N. Haag, P. Halldén, F. Hellberg, A. I. S. Holm, H. A. B. Johansson, A. Källberg, G. Källersjö, M. Larsson, S. Leontein, L. Liljeby, P. Löfgren, B. Malm, S. Mannervik, M. Masuda, D. Misra, A. Orbán, A. Paál, P. Reinhed, K.-G. Rensfelt, S. Rosén, K. Schmidt, F. Seitz, A. Simonsson, J. Weimer, H. Zettergren, H. Cederquist, Rev. Sci. Instrum. 82, 065112 (2011) and references therein.
[9] A. Kruglov, L. Weissman, P. Van den Bergh, M. Huyse, P. Van Duppen, Nucl. Phys. A 701, 193c (2002).

[10] A. Kruglov, L. Weissman, P.Van den Bergh, A. Andreyev, M. Huyse, P.Van Duppen, Nucl. Instrum. Methods Phys. Res. A 441, 595 (2000).

[11] L. Badano, O. Ferrando, M. Pezzetta, G. Molinari, Proc. DIPAC 2003, Eds: A. Peters, V.R.W. Schaa, CT09, Mainz, Germany.

[12] P.N. Ostroumov, P. Billquist, M. Portillo and W.Q. Shen, Rev. Sci. Instrum. 73, 56 (2002).

[13] L. Brännholm, S. Das, A. Källberg, A. Paal, A. Simonsson, J. Sjöholm, H. Danared, D. Reistad, in: Proc. 2nd Int. Particle Accelerator Conf. (IPAC 2011), San Sebastian (Spain), Ed: C. Petit-JeanGenaz, San Sebastián 2011.

[14] S. Das, A. Källberg, J. Harasimowicz, in: 10th European Workshop on Beam Diagnostics and Instrumentation for Particle Accelerators, DIPAC 2011, Hamburg (Germany), Eds: M. Marx, J. Chrin, I. Martin, R. Mueller, V.R.W. Schaa, Hamburg 2011.

[15] J. Harasimowicz, private communication. 